\title{
AN IRIS-INCLUSION COMPLICATION
}

\author{
BY
}

\author{
LT.-Col. H. HeRBERT, I.M.S.(retd.)
}

BRIGHTON

I HAVE to record a case of exceptional interest resembling sympathetic ophthalmitis, following an iris-inclusion operation for advanced chronic glaucoma.

Iritis set in ten days after the operation, and soon involved the other eye also. It persisted in both eyes for several months practically untreated, spreading to the ciliary body and the deeper layers of the cornea of the operated eye. But the case differed from traumatic sympathetic ophthalmitis in some important particulars :

1. Both eyes had repeatedly suffered from iritis before, as a complication of recurring subacute rheumatic fever.

2. The post-operative inflammation was evidently of exactly the same rheumatic type as the earlier attacks, though lasting considerably longer in both eyes, and being distinctly severer in the operated eye than ever before. There was a sudden onset with precisely the same pain as before, extending backwards with tenderness of the scalp, early ciliary congestion and some tendency to lacrymation. There was slight early turbidity of aqueous, with grey lines on the back of the cornea, and later, at one time there was considerable loose exudate in the anterior chamber of the operated eye, with coarse deep blood-vessels in the semi-opaque cornea. The course of events was quite different from that of recognized sympathetic disease, with its quiet gradual onset, slight subjective symptoms, keratic precipitates or early plastic exudate.

3. More important, there was an absolutely free period of ten days before the onset in the operated eye, emphasized by a very exceptionally wide dilatation of the pupil-a legacy of somewhat overfree use of cocain, adrenalin and atropin immediately before operation, but fully maintained afterwards by the routine single daily instillation of atropin.

I should like to emphasize the fact that this is a very different condition from that of low grade ectogenous infection. In the latter, though pain and congestion tend to be late in development, in my experience it is quite impossible to dilate the pupil at all fully, however early one attempts to do so after operation. One per cent. atropin drops used to the maximum from the first day after operation-nine or ten daily instillations--induce only partial mydriasis, and the pupil and coloboma, on careful examination with a loupe, fail to present a quite clean appearance from the beginning. 
Supplementary details. The patient is a man, aged 68 years, with a history of repeated attacks of rheumatic fever during the last twenty years. The first attack lasted, with relapses, nine months; but it is said that there have been quite half a dozen more or less distinct periods of joint affection, evidently subacute. He has been invalided continuously .through heart trouble. At first the eyes escaped, but since 1911 they are said to have been involved on each occasion as the joint affections were subsiding--always both eyes, but not both starting at the same, or becoming equally acutely inflamed at the same time. Finally in 1923, the eyes were attacked alone, without any joint disturbance. Thus there was apparently a steadily increasing tendency to localization in the eyes. The eye condition was treated on the first occasion at the Sussex County Hospital; afterwards by his panel doctor only, as the patient was practically confined to his house. Each attack of eye inflammation lasted possibly two months. The inflammation cannot have been very severe, since in one eye, the operated eye, I found no traces of it left. In the other, the right eye, there was a broad posterior iris-synechia and a pigment spot on the lens.

There is no evidence that the iritis had any influence in inducing the glaucoma. The latter had the features of pure chronic simple glaucoma of both eyes. The right eye had been quite blind for two and a half years. The left, upon which I operated, was also blind except for a characteristic small patch of vision in the outer field, where one or two fingers could be counted at a distance of about six inches. The irides were bright, and the media perfectly clear, allowing the deeply-cupped optic discs to be seen easily. The tension was +2 in each eye, but gave way distinctly to eserin, under the use of which for a fortnight before operation the vision of the left eye improved slightly, to counting fingers at over one foot distance.

Supporting this assumption of the absence of any aetiological connection between the previous iritis and the glaucoma, the interesting fact may be noted that throughout the months of post-operative inflammation, the tension of both eyes was definitely subnormal.

The iris-inclusion operation was performed quite satisfactorily and uneventfully on July 4, 1925, at the Worthing Hospital. A week later the vision had fallen back to the original counting of fingers at six inches. There was a slight return of plus tension, probably connected with the extreme dilatation of pupil, above mentioned. Two days later eserin instillation was hegun again on this account, and continued for five days, i.e., until I saw the patient again. The vision was then reduced to hand movements only; the tension was down, and there was iritis. So atropin was again ordered, and the patient allowed to go home. I saw him again a week later, but after this he was in the hands of his panel doctor, there being practically no prospect of any recovery of vision. I lost touch with the case till November. Both eyes were then inflamed, the left showing the exudate and vascular cornea above mentioned. The patient had had no constitutional treatment. I suggested that salicylate should be given, as on all former occasions, but the suggestion was not accepted. 
To exclude the bare possibility of a gonococcal origin of the case, Mr. F. H. Lawson, Medical Officer of the V.D. Clinic, Brighton, kindly visited the patient. He took specimens of prostatic secretion, urine and blood-one of the latter for gonococcal complement deviation test, and also, as a matter of routine, another for a Wassermann test. All the tests were negative.

The eyes remained slightly injected and watery, and the left one tender on pressure, until finally in February, 1926, "rheumatic medicine," presumably salicylate, was given. I saw the patient a month later, and found the operated eye at last perfectly quiet, and the tension felt normal. The cornea was opaque and the vision mere perception of light. In the other eye the tension was raised again definitely, with exclusion of the pupil.

Remarks. My personal interest in the case was enormously stimulated by the fact that this has been the only experience that had caused the slightest wavering in a slowly acquired belief that iris-inclusion must eventually become the chief operative treatment of at least the severer primary glaucomas. This faith predicates a reasoned confidence in the feasibility of total and certain exclusion of ectogenous infection. A single unexplained case of sympathetic ophthalmitis in my own practice would be sufficient to destroy the belief.

Recent experience has tended greatly to strengthen the conviction that true traumatic sympathetic disease represents an invasion of the exciting eye by external organisms. One may instance the almost complete elimination of the disease in the great war "by increased skill in the application of antiseptic principles . . . in spite of the fact that ophthalmic surgery has become more conservative."

My own feeling of security, as regards the prevention of ectogenous infection, is based primarily on cataract work in India, which attained dimensions sufficient to afford the needed sense of reliability. Both Colonel Elliot and I were able, with the help of measured perchloride irrigation of the conjunctiva, entirely to avoid primary suppurations, while greatly reducing lower grade infections, in our final periods of Indian work (see Trans. Ophthal. Soc., U.K., 1914, pp. 46-8, 280-1).

From this degree of success in dealing with the large and often imperfectly covered wounds of cataract extraction, it is not unreasonable to expect complete success in the exclusion of surface organisms from the small subconjunctival wounds that suffice in glaucoma work. So far as I know, this expectation has been realized in all my iris-inclusion operations since measured irrigation was adopted for them (Trans. Ophthal. Soc., U.K., 1903, p. 327). It would be strange, after so many years of freedom from ordinary septic invasions, now to get a quite unexplained instance. of the less common sympathetic disease. More particularly does 
this apply since the full two minutes' irrigation with 1 in 3,000 sublimate solution is now practically always employed.

For this reason, in addition to the three given on page 380, 1 conclude that the double complication here reported was endogenous-a recurrence of the old rheumatic inflammation, excited and, in the operated eye, aggravated in some way by the operative interference.

Analyzing the post-operative sequence of events, it was remarkable that the actual operation seemed to have troubled the eye very little, if at all. The sudden onset of iritis when eserin instillations were resumed nine days after the operation suggests that the sudden flooding of the blood-vessels of the iris, after a period of very marked compression by extreme dilatation of the pupil, may have set free, or rejuvenated, encysted organisms left in the iris from previous rheumatic attacks.

It may be noted that the drag upon the iris was quite localized in this case. The impaction was exceptionally small, since all of the iris except that normally situated quite close to the sclerocorneal wound was withdrawn from the wound by the very marked dilatation of the pupil. It is questionable whether the same result might not have happened from any operation leaving the iris unimpacted, as occurs in endogenous infection (of the operated eye) following cataract extraction.

The value of the case lies in the fact that while it establishes clearly an endogenous post-operative complication liable to be mistaken for true traumatic sympathetic disease, it also supplies distinctive features that may be of use in the recognition of other cases of the same nature, perhaps not so fully differentiated.

It will be interesting to note whether in all such cases the affection of the operated eye proves to be, as in this instance, severer than in the fellow eye.

The experience is altogether too exceptional to act as a deterrent in considering the treatment of glaucoma by iris-inclusion, except in the rare cases where there may be, as in this patient, a history of previous recurrent iritis. Rheumatic fever is only one of the rarer causes of iritis, and the vulnerability of this patient to the disease was evidently very exceptional; and it was a rare chance that the rheumatic inflammation happened to fall on eyes predisposed to glaucoma.

On a few occasions I have used irid-encleisis for plus tension left by former iritis or irido-cyclitis of common type. The operations were ineffectual, but there was no re-lighting of the old inflammation.

Operative Considerations. Until such endogenous complications are clearly recognizable, it is impossible to get a true perspective regarding treatment by iris-incarceration. Granted that true 
sympathetic ophthalmitis is due to an external organism, it is not right that the fear of the disease should debar the patient from the promise of benefits held out apparently only by iris-incarceration. The advantages apparently realizable are uniformly permanent and yet moderate drainage, elimination of hypotony and of the conjunctival bleb, also of the dangers of a large incision and of delayed re-formation of the anterior chamber.

It is the surgeon's business to exclude all possibility of surface infection, and there is sufficient evidence that it can be done. Unless exceptionally reliable culture tests are available, some proved means of clearing away conjunctival bacteria should always be used. The only means with which I am personallv acquainted is the maximal perchloride irrigation that has stood the test of a quarter of a century of practical experience in India. This, with swabbing of the lid margins by the same solution, causes a little conjunctival and lid swelling. Conjunctival injection is controlled during operation by adrenalin. There is a variable degree of smarting for a few hours afterwards, and there is often a little mucoid discharge later. But it never does any harm, and I have never found that the patients object to it. They infinitely prefer it to running the slightest risk.

Also the method of operating should be as subconjunctival as possible. I should like merely to mention a technique that promises particularly well. Dissatisfied with imperfect subconjunctival drainage in operating with the triangular keratome, I have lately gone back to the narrow tapering glaucoma knife. The sclerocorneal incision is made after sliding the conjunctiva. The only opening in the latter is vertical or oblique, $4-5 \mathrm{~mm}$. long, in the upper outer quadrant of the eye, $4-5 \mathrm{~mm}$. away from the cornea. And the subconjunctival drainage up to date appears perfect. But I have only done a few of these operations, and it is too early to speak of the final results.

\section{AN INTERESTING EXAMPLE OF HEREDITARY DISLOCATION OF THE LENS OCCURRING IN FOUR SUCCESSIVE GENERATIONS}

BY

Elizabeth Pace Cameron, M.B., Ch.B., D.P.H. CARDIFF

THE following example of hereditary dislocation of the lens occurring in four successive generations, appears to be of sufficient interest to be worth recording. There have been fourteen persons affected of whom only one was a male. Five have had to be trained in institutions for the blind. In every case, both lenses have been 